\title{
A METHOD OF SYMMETRIZATION AND APPLICATIONS. II
}

\author{
BY
}

\author{
DOV AHARONOV AND W. E. KIRWAN( $\left.{ }^{1}\right)$
}

\begin{abstract}
In this paper we make use of a new method of symmetrization introduced in [1] to study various covering properties of univalent functions. More specifically, we introduce a generalization of a classical problem raised by Fekete and give a partial solution.
\end{abstract}

1. Introduction. Let $D$ here and throughout this paper denote a plane domain containing the origin with mapping radius (inner conformal radius) 1 at 0 ([3] is a good reference for definitions in the paper). Let $l_{k}$ denote the linear measure of $D \cap\{w \mid \arg w=2 k \pi / n\}$ where $k$ denotes an integer and $1 \leq k \leq n$. M. Fekete conjectured that the minimum value of $\Pi_{k=1}^{n} l_{k}$ as $D$ ranges over the class of all simply connected domains with mapping radius 1 at 0 (in the sequel we write $r(D, 0)=1)$ is $1 / 4$, and that the extremal domain is the complement of $n$ radial slits extending to $\infty$ along the rays arg $w=2 k \pi / n$ with the tip of each slit being at a distance $\sqrt[n]{1 / 4}$ from the origin. M. Marcus [5] proved this conjecture using a generalization of a method of symmetrization introduced by G. Szegö [8]. In this note we consider a natural generalization of the above problem. Let

$$
0 \leq \sigma_{1} \pi<\sigma_{2} \pi<\cdots<\sigma_{n} \pi \leq 2 \pi
$$

be $n$ angles and denote by $l_{k}$ the linear measure of $D \cap\left\{w \mid \arg w=\sigma_{k} \pi\right\}$. What is the minimum value of $\Pi_{k=1}^{n} l_{k}$ over the class of domains $D$ with $r(D, 0)=1$ and can one characterize the shape of the extremal domain? It is not difficult to show that the extremal domain is the complement of radial slits along the rays arg $w=$ $\sigma_{k} \pi$. Are there choices of the $\sigma_{k}$ other than $\sigma_{k} \pi=2 k \pi / n$ (where the problem reduces to the Fekete problem mentioned above) when the tips of the slits are equidistant from the origin? In this note we answer this question in the affirmative. Further, when the $\sigma_{k} \pi$ are restricted so that

$$
\sigma_{k} \pi=2 \pi-\sigma_{n-k+1} \pi \quad(1 \leq k \leq n),
$$

which we will always assume in the sequel, we determine an expression whose

Received by the editors May 25, 1971.

AMS 1969 subject classifications. Primary 3040; Secondary 3041, 3042, 3052.

Key words and phrases. Univalent functions, conformal mapping, mapping radius, radial slits, covering theorems.

(1) The work of this author was supported in part by National Science Foundation Grant GP 12547. 
extreme values give the minimum of $\Pi_{k=1}^{n} l_{k}$ over the class of domains $D$. For certain values of the $\sigma_{k}$, this expression is closely related to the Vandermonde determinant.

The main tool in this paper is a method of symmetrization for plane domains introduced in [1]. This method of symmetrization generalizes the method of symmetrization introduced by Marcus and is defined as follows. Let $G$ be a plane domain containing 0 . If the disk $|z|<\rho$ is contained in $G$ define

$$
L_{\rho}(\phi)=\int_{E} \frac{d r}{r}
$$

where $E=E_{\rho}(\phi)$ is the intersection of $D$ with $\{z|| z \mid \geq \rho$, $\arg z=\phi\}$, and

$$
R(\phi)=\rho \exp L_{\rho}(\phi) .
$$

$R(\phi)$ is of course independent of $\rho$. Let $A=\left\{\alpha_{k}\right\}_{k=1}^{n}$ and $B=\left\{\beta_{k}\right\}_{k=1}^{n}$ be two sequences of real numbers with $\left|\alpha_{k}\right|=1$. Define

$$
R^{(n)}(\phi)=R^{(n)}(\phi, A, B)=\left[\prod_{k=1}^{n} R\left(\alpha_{k} \phi+\beta_{k}\right)\right]^{1 / n}
$$

The $P_{n}[A, B]=P_{n}$ symmetrized domain of $G$, denoted $P_{n} G$, is defined by

$$
P_{n} G=\left\{z \mid z=r e^{i \phi}, 0 \leq r<R^{(n)}(\phi), 0 \leq \phi<2 \pi\right\} \text {. }
$$

Notes. 1. With the choice $\alpha_{k}=1, \beta_{k}=2 k \pi / n, P_{n}$ symmetrization reduces to the method of symmetrization defined by Marcus [5].

2. $P_{n} G$ is a simply connected domain, starlike with respect to 0 .

3. If $l(\phi)$ denotes the linear measure of $D \cap\{w \mid \arg w=\phi\}$, it is not hard to show that $l(\phi) \geq R(\phi)$ and equality holds if and only if $D \cap\{w \mid \arg w=\phi\}$ is connected.

4. It was shown in [1] that $r(G, 0) \leq r\left(P_{n} G, 0\right)$.

5. The choice $n=2, \alpha_{1}=1, \alpha_{2}=-1$ and $\beta_{1}=0=\beta_{2}$ is particularly important in this paper. In this case the symmetrized domain is symmetric about the real axis and the distances from 0 to the boundary along the two rays $\arg w= \pm \phi$ $(0 \leq \phi<2 \pi)$ are equal.

\section{The main theorems.}

Theorem 1. Let $D$ denote a plane domain containing 0 with $r(D, 0)=1$. Let $n=2 m$ be an even integer and let $\left\{\sigma_{k} \pi\right\}_{k=1}^{n}$ satisfy (1.1) and (1.2). If $l_{k}$ denotes the linear measure of $D \cap\left\{w \mid \arg w=\sigma_{k} \pi\right\}$ then 


$$
\prod_{k=1}^{n} l_{k} \geq \min _{0 \leq a_{i} \leq 1} \frac{1}{4^{n-1}} \prod_{k=0}^{m} \eta_{k}^{-\eta} \prod_{0 \leq k<j \leq m}\left|a_{j}-a_{k}\right|^{-\eta_{j}-\eta_{k}}
$$

where the minimum is taken over all sequences $0=a_{0} \leq a_{1} \leq \cdots \leq a_{m}=1,2 \sigma_{k}=$ $\sum_{j=0}^{k-1} \eta_{j}$ for $1 \leq k \leq m$ and $\sum_{j=0}^{m} \eta_{j}=2$. The inequality is sharp.

Proof. Let $D$ be a domain satisfying the hypothesis of the theorem. We symmetrize $D$ using the special form of $P_{n}$ symmetrization discussed in Note 5 of $\S 1$. Let $D^{*}$ denote the symmetrized domain and $l_{k}^{*}$ the linear measure of $D^{*} \cap$ $\left\{w \mid \arg w=\sigma_{k} \pi\right\}$. Clearly $l_{k}^{*}=l_{n+1-k}^{*}$. It follows from Note 3 of $\$ 1$ that

$$
l_{k}^{*}=\left[R\left(\sigma_{k} \pi\right) R\left(\sigma_{n+1-k} \pi\right)\right]^{1 / 2} \leq\left[l_{k} l_{n+1-k}\right]^{1 / 2}
$$

and consequently

$$
\prod_{k=1}^{n} l_{k}^{*} \leq \prod_{k=1}^{n} l_{k}
$$

Also, $\lambda=r\left(D^{*}, 0\right) \geq r(D, 0)=1$. Let $\hat{D}=\left\{w \mid \lambda w \in D^{*}\right\}$ and $\hat{l}_{k}$ the linear measure of $\hat{D} \cap\left\{w \mid \arg w=\sigma_{k} \pi\right\}$. Then $r(\hat{D}, 0)=1$ and so if $D$ is an extremal domain for this problem then, by (2.2),

$$
\prod_{k=1}^{n} l_{k} \leq \prod_{k=1}^{n} \hat{l}_{k} \leq \prod_{k=1}^{n} l_{k}^{*} \leq \prod_{k=1}^{n} l_{k}
$$

Hence there exists an extremal domain for this problem that is starlike with respect to 0 and satisfies $l_{k}=l_{n+1-k}$ for $1 \leq k \leq n$. Using the monotonicity of $r(D, 0)$ (i.e., $r\left(D_{1}, 0\right) \leq r\left(D_{2}, 0\right)$ if $\left.D_{1} \subset D_{2}\right)$ it follows that this extremal domain is the complement of slits extending to $\infty$ along the rays $\arg w=\sigma_{k} \pi$ with the distance from 0 to the tip of the $k$ th slit being $l_{k}$. Since $\sigma_{k} \pi=2 \pi-\sigma_{n+1-k} \pi$ and $l_{k}=l_{n+1-k}$, it follows from the Herglotz representation for starlike functions [2, p. 529] that a function of the form

$$
f(z)=z(1-z)^{-\eta_{0}}(1+z)^{-\eta_{m}} \prod_{k=1}^{m-1}\left[\left(1-\exp \left(i \theta_{k}\right) z\right)\left(1-\exp \left(-i \theta_{k}\right) z\right)\right]^{-\eta_{k} / 2}
$$

where $2 m=n, 0 \leq \theta_{k} \leq 2 \pi, 2 \sigma_{k}=\sum_{j=0}^{k-1} \eta_{j}(0 \leq k \leq m)$ and $\sum_{0}^{m} \eta_{k}=2$ maps the unit disk $U$ onto this extremal domain. Thus an extremal domain for this problem is obtained by minimizing $\Pi_{k=1}^{n} l_{k}$ over domains obtained from functions of the form (2.3) for varying $\theta_{k}$. With $z=e^{i \phi}, y=\sin ^{2}(\phi / 2), a_{0}=0, a_{m}=1$ and $\sin ^{2}\left(\theta_{k} / 2\right)=$ $a_{k}$ for $1 \leq k \leq m-1,(2.3)$ can be replaced by 


$$
\left|f\left(e^{i \phi}\right)\right|^{2}=\frac{1}{16} \prod_{k=0}^{m}\left|y-a_{k}\right|^{-\eta_{k}}
$$

The values of $\phi$ that correspond to the tips of the slits on the boundary of $f(U)$ are determined from the $m$ roots of the equation

$$
\sum_{k=0}^{m} \frac{\eta_{k}}{y-a_{k}}=0 \quad\left(y=\sin ^{2} \frac{\phi}{2}\right)
$$

If $z_{1}, \cdots, z_{n}$ denote points on $|z|=1$ which are the pre-images of the tips of these slits, then since $l_{k}=l_{n+1-k}$ and $\sigma_{k} \pi=\sigma_{n+1-k} \pi,\left|f\left(z_{k}\right)\right|=\left|f\left(z_{n+1-k}\right)\right|$ and consequently

$$
\begin{array}{rlrl}
\prod_{k=1}^{n} l_{k} & =\prod_{j=1}^{m}\left|f\left(z_{j}\right)\right|^{2} & \left(z_{j}=\exp \left(i \phi_{j}\right)\right) \\
& =\frac{1}{16^{m}} \prod_{j=1}^{m} \prod_{k=0}^{m}\left|y_{j}-a_{k}\right|^{-\eta_{k}} \quad\left(y_{j}=\sin ^{2} \frac{\phi_{j}}{2}\right) \\
& =\frac{1}{16^{m}} \prod_{k=0}^{m} \prod_{j=1}^{m}\left|y_{j}-a_{k}\right|^{-\eta_{k}} . &
\end{array}
$$

If $y_{1}, \cdots, y_{m}$ denote the roots of (2.5), then for each fixed $k, 0 \leq k \leq m$, $y_{1}-a_{k}, \cdots, y_{m}-a_{k}$ are the roots of $\sum_{j=0}^{m} \eta_{j} /\left(x-\left(a_{j}-a_{k}\right)\right)=0$. Hence

$$
\prod_{j=1}^{m}\left|y_{j}-a_{k}\right|=\frac{1}{2} \eta_{k} \prod_{j=0 ; j \neq k}^{m}\left|a_{j}-a_{k}\right| \text {. }
$$

Substituting (2.7) in (2.6) we obtain

$$
\begin{aligned}
\prod_{k=1}^{n} l_{k} & =\frac{1}{16^{m}} \prod_{k=0}^{m}\left[\frac{1}{2} \eta_{k} \prod_{j \neq \dot{k}}\left|a_{j}-a_{k}\right|\right]^{-\eta_{k}} \\
& =\frac{1}{4^{n-1}} \cdot \prod_{k=0}^{m} \eta_{k}^{-\eta_{k}} \cdot \prod_{0 \leq k<j \leq m}\left|a_{j}-a_{k}\right|^{-\eta_{j}-\eta_{k}} .
\end{aligned}
$$

Taking the minimum of (2.8) over all $a_{k}, 0 \leq a_{k} \leq 1$, we see that the proof is complete.

Theorem 2. With the notation of Theorem 1, if $n=2 m+1$ is an odd integer then

$$
\prod_{k=1}^{n} l_{k} \geq \min _{0 \leq a_{i} \leq 1} \frac{1}{4^{n-1}} \prod_{k=0}^{m} \eta_{k}^{-\eta_{k}} \prod_{0 \leq k<j \leq m}\left|a_{k}-a_{j}\right|^{-\eta_{k}-\eta_{j}} \prod_{j=1}^{m}\left(1-a_{j}\right)^{-\eta_{j} / 2} .
$$

The inequality is sharp. 
For odd $n, n=2 m+1$, an argument similar to one used in the proof of Theorem 1 shows that a function of the form

$$
f(z)=z(1-z)^{-\eta_{0}} \prod_{k=1}^{m}\left[\left(1-z \exp \left(-i \theta_{k}\right)\right)\left(1-z \exp \left(i \theta_{k}\right)\right)\right]^{\eta_{k} / 2}
$$

maps $U$ onto the extremal domain. The equation for odd $n$ analogous to (2.4) is

$$
\left|f\left(e^{i \phi}\right)\right|^{2}=\frac{1}{16} \prod_{k=0}^{m}\left|y-a_{k}\right|^{-\eta_{k}} \quad\left(y=\sin ^{2} \frac{\phi}{2}\right) .
$$

The proof of Theorem 2 proceeds from this equation in precisely the same way as the proof of Theorem 1 from (2.4).

If $n=1$, Theorem 2 is a consequence of the Koebe- $1 / 4$ Theorem. If $n=2$, Theorem 1 gives the result that

$$
l_{2} \cdot l_{2} \geq \frac{1}{4} \frac{1}{\eta^{\eta}} \frac{1}{(2-\eta)^{2}-\eta}
$$

where $\eta=2 \sigma_{1}$. This result was previously obtained in [1] and [4]. We will now give some examples illustrating Theorems 1 and 2 for other values of $n$.

Example 1. Let $n=3$. Then $\sigma_{2} \pi=\pi, \eta_{0}+\eta_{1}=2$, and by Theorem 2 for any domain $D$ with $r(D, 0)=1$,

$$
\prod_{k=1}^{3} l_{k} \geq \min _{0 \leq a \leq 1} \frac{1}{4^{2}} \eta_{0}^{-\eta_{0}} \eta_{1}^{-\eta_{1}} a^{-2}(1-a)^{-\eta_{1} / 2}
$$

The minimum occurs when $a=4 /\left(4+\eta_{1}\right)$ and hence

$$
\prod_{k=1}^{3} l_{k} \geq \frac{1}{4^{2}} \eta_{0}^{-\eta_{0}} \eta_{1}^{-\eta_{1}}\left(\frac{4+\eta_{1}}{4}\right)^{2}\left(\frac{4+\eta_{1}}{\eta_{1}}\right)^{\eta_{1} / 2}
$$

Example 2. Let $n=4$. If $D$ is a domain with $r(D, 0)=1$, then by Theorem 1

$$
\prod_{k=1}^{4} l_{k} \geq \min _{0 \leq a \leq 1} 4^{-3}\left(\eta_{0}^{\eta_{0}}\right)^{-1}\left(\eta_{1}^{\eta_{1}}\right)^{-1}\left(\eta_{2}^{\eta_{2}}\right)^{-1} a^{-\left(\eta_{0}+\eta_{1}\right)}(1-a)^{-\left(\eta_{1}+\eta_{2}\right)}
$$

The minimum occurs when $a=\left(\eta_{0}+\eta_{1}\right) /\left(2+\eta_{1}\right)=\left(2-\eta_{2}\right) /\left(2+\eta_{1}\right)$ and hence

$$
\begin{aligned}
\prod_{k=1}^{4} l_{k} & \geq 4^{-3}\left(\eta_{0}^{\eta_{0}}\right)^{-1}\left(\eta_{1}^{\eta_{1}}\right)^{-1}\left(\eta_{2}^{\eta_{2}}\right)^{-1}\left(\frac{2+\eta_{1}}{2-\eta_{2}}\right)^{2-\eta_{2}}\left(\frac{2+\eta_{1}}{2-\eta_{0}}\right)^{2-\eta_{0}} \\
& =4^{-3} \prod_{k=0}^{2} \eta_{k}^{-\eta_{k}} \frac{\left(2+\eta_{1}\right)^{2+\eta_{1}}}{\left(2-\eta_{2}\right)^{2-\eta_{2}}\left(2-\eta_{0}\right)^{2-\eta_{0}}} .
\end{aligned}
$$


Example 3. Let $n=5$. If $D$ is a domain with $r(D, 0)=1$ then, by Theorem 2 ,

$$
\begin{aligned}
\prod_{k=1}^{5} l_{k} \geq \min _{0 \leq a \leq b \leq 1} \frac{1}{4^{4}}\left[\prod_{k=0}^{2} \eta_{k}^{-\eta_{k}}\right] a^{-\left(\eta_{0}+\eta_{1}\right)} b^{-\left(\eta_{0}+\eta_{2}\right)} \\
\cdot|a-b|^{-\left(\eta_{1}+\eta_{2}\right)}(1-a)^{-\eta_{1} / 2}(1-b)^{-\eta_{1} / 2} .
\end{aligned}
$$

The values of $a$ and $b$ that minimize (2.11) are solutions $a=u$ and $b=v$ of the equations

$$
\begin{aligned}
& \frac{\eta_{1}}{2(u-1)}+\frac{\eta_{1}+\eta_{2}}{u-v}+\frac{\eta_{0}+\eta_{1}}{u}=0, \\
& \frac{\eta_{2}}{2(v-1)}-\frac{\eta_{1}+\eta_{2}}{u-v}+\frac{\eta_{0}+\eta_{2}}{v}=0 .
\end{aligned}
$$

We multiply these equations by $u^{2}$ and $v^{2}$ respectively and add the two expressions obtaining a new equation. We then multiply by $u$ and $v$ respectively and add the two expressions obtaining a second equation. If we now subtract these two equations, the equation

$$
\left(2+(3 / 2) \eta_{1}\right) u+\left(2+(3 / 2) \eta_{2}\right) v=4
$$

results. Solving for $v$ in (2.13) and substituting in the first equation of (2.12) we obtain a quadratic equation in $u$ that has two positive roots $0<u_{1} \leq u_{2}$. Similarly substituting for $u$ in the second equation of (2.12) we obtain a quadratic equation that has two positive roots $0<v_{2} \leq v_{1}$. It follows from (2.13) that only $\left(u_{1}, v_{1}\right)$ and $\left(u_{2}, v_{2}\right)$ can be solutions of (2.12). We claim that $a=u_{1}$ and $b=v_{1}$ are the values that give the minimum in (2.11). First we note that since the minimum in (2.11) is attained, there is a solution $(u, v)$ of (2.11) with $u<1$ and $u \leq v$, and hence at least one of the pairs $\left(u_{1}, v_{1}\right)$ and $\left(u_{2}, v_{2}\right)$ satisfies this inequality. This implies that $u_{1} \leq v_{1}$. Suppose that $u_{1} \leq u_{2}<v_{2} \leq v_{1}$ and $u_{2}<1$. Then $v_{1}-u_{1}>v_{2}-u_{2}$. However, it is not hard to show that this is incompatible with the first equation of (2.12). Thus $\left(u_{1}, v_{1}\right)$ is the only one of the two pairs with $u_{1} \leq v_{1}$ and $u \leq 1$ (except for the trivial case $u_{1}=u_{2}$ ) and consequently gives the minimum of (2.11).

If, in Theorem 1, $\sigma_{k} \pi=2 k \pi /(n+2)(1 \leq k \leq m)$ and $\sigma_{m+k}=\sigma_{k} \pi+\pi=$ $2(m+k+1) \pi /(n+2)(1 \leq k \leq m)$, then $\eta_{0}=\eta_{1}=\cdots=\eta_{m}=2 /(m+1)$. In this case the determination of the minimum of $(2.1)$ is equivalent to maximizing the Vandermonde determinant $V\left(a_{0}, a_{1}, \cdots, a_{m}\right)$ over the region $0 \leq a_{0} \leq a_{1} \leq \cdots \leq a_{m} \leq 1$. The latter problem was solved by Stielties [7] and Schur [6]. Using their result we have the following theorem.

Theorem 3. Let $n=2 m$ and $\sigma_{k} \pi=2 k \pi /(n+2)$ for $1 \leq k \leq m$. If $D$ is a domain with $r(D, 0)=1$ then 


\section{$\prod_{k=1}^{n} l_{k}$}

$$
\geq \frac{1}{2^{n-2}}\left(\frac{m+1}{2}\right)^{2}\left[\frac{3^{3} \cdot 5^{5} \cdots(2 m-1)^{2 m-1}}{2^{2} \cdot 3^{3} \cdots(m+1)^{m+1} \cdot 2^{2} \cdot 3^{3} \cdots(m-1)^{m-1}}\right]^{2 /(m+1)}
$$

The inequality is sharp.

Proof. Since $\eta_{0}=\eta_{1}=\cdots=\eta_{m}=2 /(m+1)$, we have from Theorem 1 that

$$
\prod_{k=1}^{n} l_{k} \geq \frac{1}{4^{n-1}}\left(\frac{m+1}{2}\right)^{2} \min _{0 \leq a_{i} \leq 1}\left[\prod_{0 \leq k<j \leq m}\left|a_{j}-a_{k}\right|\right]^{-4 /(m+1)} .
$$

As mentioned above, Stieltjes and later Schur proved that

$$
\begin{aligned}
\max _{0 \leq a_{1} \leq \cdots \leq a_{m} \leq 1} & \prod_{0 \leq i<k \leq m}\left|a_{i}-a_{k}\right| \\
& =2^{-m(m+1) / 2}\left[\frac{2^{2} \cdot 3^{3} \cdots(m+1)^{m+1} \cdot 2^{2} \cdot 3^{3} \cdots(m-1)^{m-1}}{3^{3} \cdot 5^{5} \cdots(2 m-1)^{2 m-1}}\right]^{1 / 2}
\end{aligned}
$$

and the proof is complete.

Corollary. Let $m$ be a positive integer and $\sigma_{k} \pi=2 k \pi /(m+1)$ for $1 \leq k \leq m$. If $D$ is a domain with $r(D, 0)=1$ then

$$
\begin{aligned}
& \prod_{k=1}^{m} l_{k} \\
& \quad \geq \frac{1}{4^{m-1}}\left(\frac{m+1}{2}\right)^{2}\left[\frac{3^{3} \cdot 5^{5} \cdots(2 m-1)^{2 m-1}}{2^{2} \cdot 3^{3} \cdots(m+1)^{m+1} \cdot 2^{2} \cdot 3^{3} \cdots(m-1)^{m-1}}\right]^{2 /(m+1)}
\end{aligned}
$$

and the inequality is sharp.

Proof. Let $D_{0}$ denote an extremal domain for this problem. Using an argument similar to one used in the proof of Theorem 1 , it follows that $D_{0}$ is the complement of radial slits along the rays arg $w=2 k \pi /(m+1)(1 \leq k \leq m)$ and that $D_{0}$ is symmetric with respect to the real axis. Let $f_{0}(z)$ map $U$ onto $D_{0}$ and set $g_{0}(z)$ $=\left[f_{0}\left(z^{2}\right)\right]^{1 / 2}$ for $z$ in $U \cdot g_{0}(z)$ maps $U$ onto a domain whose complement consists of radial slits along the $n=2 m$ rays $\arg w=2 k \pi /(2 m+2)=2 k \pi /(n+2)$ for $1 \leq k \leq m$ and $\arg w=2(k+1) \pi /(n+2)$ for $m+1 \leq k \leq n$. If $g_{1}(z)$ maps $U$ onto the extremal domain of Theorem 3 with $n=2 m$ then

$$
\prod_{k=1}^{n} l_{k}\left(g_{0}\right) \geq \prod_{k=1}^{n} l_{k}\left(g_{1}\right)
$$


$g_{1}(U)$ is symmetric with respect to the real axis. In addition we may assume $g_{1}(z)$ is an odd function. To see that this is the case, we symmetrize $g_{1}(U)$ using (1.3) with

$$
R^{(2)}(\phi)=[R(\phi) \cdot R(\phi+\pi)]^{1 / 2}
$$

The symmetrized domain $D^{*}$ is symmetric with respect to the origin and, because of the nature of $g_{1}(U)$, with respect to the real axis. If $g_{2}(z)$ maps $U$ conformally onto $D^{*}$ with $g_{2}(0)=0$ and $g_{2}^{\prime}(0)>0$ then

$$
\prod_{k=1}^{n} l_{k}\left(g_{2}\right)=\prod_{k=1}^{n} l_{k}\left(g_{1}\right) \text {. }
$$

Now $g_{2}^{\prime}(0)=r\left(D^{*}, 0\right) \geq r\left(g_{1}(U), 0\right)=1$ and $g_{1}(z)$ is an extremal function for (2.14). It follows that $g_{2}^{\prime}(0)=1$, for otherwise $\left(1 / g_{2}^{\prime}(0)\right) g_{2}(z)$ would map $U$ onto a domain with mapping radius 1 and by $(2.16)$ contradict the fact that $g_{1}(z)$ is an extremal function. Since we may assume $g_{1}(z)=g_{2}(z)$ and hence odd, $g_{1}(z)=$ $\left[f_{1}\left(z^{2}\right)\right]^{1 / 2}$ where $f_{1}(z)$ maps $U 1-1$ onto the complement of radial slits along the rays $\arg w=2 k \pi /(m+1)$. Also, for $1 \leq k \leq m$,

$$
\begin{aligned}
l_{k}\left(f_{i}\right) & =\left[l_{k}\left(g_{i}\right)\right]^{2} \quad(i=0,1) \\
& =l_{k}\left(g_{i}\right) \cdot l_{n+1-k}\left(g_{i}\right) .
\end{aligned}
$$

Therefore from (2.15) we have

$$
\prod_{k=1}^{m} l_{k}\left(f_{1}\right)=\prod_{k=1}^{n} l_{k}\left(g_{1}\right) \leq \prod_{k=1}^{n} l_{k}\left(g_{0}\right)=\prod_{k=1}^{m} l_{k}\left(f_{0}\right) .
$$

Thus $f_{1}$ is extremal function and the proof is complete.

3. Shape of the extremal domain. The extremal domain for $(2.10)$ is the complement of radial slits extending to infinity along the rays $\arg w=\sigma_{1} \pi$ and $\arg w=2 \pi-\sigma_{1} \pi$ with the tips of the slits being equidistant from the origin. As was shown by Marcus, if $\sigma_{k} \pi=2 k \pi / n$ for $1 \leq k \leq n$, then the extremal domain again has the property that the complement consists of radial slits whose tips are equidistant from the origin. We will now examine each of the examples of the previous section to see under what conditions on the $\sigma_{k} \pi$ the extremal domains of these examples have this property.

Example 1. In this case $n=3$. We will show that the tips of the slits on the boundary of the extremal domain are equidistant from the origin only if $\sigma_{1} \pi=\pi / 3$, $\sigma_{2} \pi=\pi$ and $\sigma_{3} \pi=5 \pi / 3$, i.e. when the extremal problem (2.1) reduces to the one considered by Fekete and Marcus.

When $n=3$, we have from (2.9) that 


$$
\left|f\left(e^{i \phi}\right)\right|^{2}=(1 / 16) y^{-\eta} 0|y-a|^{-\eta} 1 \quad\left(a=a_{1}\right) .
$$

From Example 1 of $\$ 2$ we have that the value of $a$ which yields the extremal function is

$$
a=4 /\left(4+\eta_{1}\right)
$$

It follows from the symmetry of the extremal function that the tip of the slit along the ray $\arg w=\pi$ is the image of the point $z=-1$. The tips of the other two slits are the images of $\exp \left(i \phi_{1}\right)$ and $\exp \left(-i \phi_{1}\right)$ where $\sin ^{2}\left(\phi_{1} / 2\right)=y=2 \eta_{0} /\left(4+\eta_{1}\right)$.

The tips of the slits are equidistant from the origin if and only if $|f(-1)|^{2}=$ $\left|f\left(\exp \left(i \phi_{1}\right)\right)\right|^{2}$ which by (3.1) and (3.2) is equivalent to $4 \eta_{0}^{\eta}{ }^{0} \eta_{1}^{\eta} 1\left(4+\eta_{1}\right)^{-2}=$ $\eta_{1}^{\eta_{1}}\left(4+\eta_{1}\right)^{-\eta_{1}}$ or

$$
4\left(\eta_{0} /\left(6-\eta_{0}\right)\right)^{\eta} 0=1 \quad\left(\eta_{0}+\eta_{1}=2\right) .
$$

$\eta_{0}=2$ and $\eta_{0}=2 / 3$ are roots of this equation. $\eta_{0}=2 / 3$ corresponds to $\sigma_{1} \pi=$ $\pi / 3$ and the slits are known to be equidistant from the origin in this case. It remains to show that this equation has no other solution for $0<\eta_{0}<2$. If there was another solution then the derivative with respect to $\eta_{0}$ of the left-hand side of (3.3) would vanish twice on $0<\eta_{0}<2$. An easy calculation shows that this is impossible.

Example 2. In this case we will show that the slits on the boundary of the extremal domain can be equidistant from the origin only if $\eta_{0}=\eta_{2}$, i.e. only if $\sigma_{3} \pi=\sigma_{1} \pi+\pi$ and $\sigma_{4} \pi=\sigma_{2} \pi+\pi$. From (2.9) we have that the extremal function $f(z)$ satisfies

$$
\left|f\left(e^{i \phi}\right)\right|^{2}=(1 / 16) y^{-\eta} 0|y-a|^{-\eta} 1|1-y|^{-\eta_{2}} \quad\left(y=\sin ^{2}(\phi / 2)\right),
$$

where, by Example 2 of $\S 2, a=\left(2-\eta_{2}\right) /\left(2+\eta_{1}\right)$. If $\exp \left(i \phi_{1}\right), \exp \left(-i \phi_{1}\right)$, $\exp \left(i \phi_{2}\right)$ and $\exp \left(-i \phi_{2}\right)$ denote the points on $|z|=1$ that are mapped by $f(z)$ onto the tips of slits on the boundary of the extremal domain, then $y_{1}=\sin ^{2}\left(\phi_{1} / 2\right)$ and $y_{2}=\sin ^{2}\left(\phi_{2} / 2\right)$ are the solutions of

$$
\eta_{0} / y+\eta_{1} /(y-a)-\eta_{2} /(1-y)=0
$$

It follows from (3.4) that for the extremal function $\left|y_{1}-a\right|=\left|y_{2}-a\right|$ and hence $\left|f\left(e^{i \phi_{1}}\right)\right|^{2}=\left|f\left(e^{i \phi_{2}}\right)\right|^{2}$ if and only if $y_{1}^{\eta_{0}}\left(1-y_{1}\right)^{\eta_{2}}=y_{2}^{\eta_{0}}\left(1-y_{2}\right)^{\eta_{2}}$. Thus the slits are equidistant from the origin for the extremal domain if and only if

$$
\left(y_{1} / y_{2}\right)^{\eta} 0=\left(\left(1-y_{2}\right) /\left(1-y_{1}\right)\right)^{\eta} 2 \text {. }
$$

From (3.4), $y_{1}$ and $y_{2}$ are roots of a quadratic equation $y^{2}-b y+c=0$. The discriminant, $d^{2}$, of this equation is $d^{2}=2 \eta_{1}\left(2-\eta_{0}\right)\left(2-\eta_{2}\right) /\left(2+\eta_{1}\right)^{2}$. Consequently 
(3.5) is equivalent to

$$
((b+d) /(b-d))^{\eta} 0=((2-b+d) /(2-b-d))^{\gamma^{\prime}} 2 .
$$

As an equation in the variable $d, d=0$ is a solution of (3.6). If $\eta_{0} \neq \eta_{2}$ we claim that this equation has no other solution (and hence that the slits on the boundary of the extremal domain are not equidistant from the origin). Indeed if there was another solution, then the derivative with respect to $w$ of

$$
G(w)=\eta_{0} \log \frac{b+w}{b-w}-\eta_{2} \log \left(\frac{2-b+w}{2-b-w}\right)
$$

would vanish on $0<w<d$. A rather lengthy but elementary calculation shows that $d<b, d<2-b$ and

$$
G^{\prime}(w)=\frac{8\left(\left(\eta_{0}-\eta_{2}\right) /\left(2+\eta_{1}\right)\right)\left[d^{2}-w^{2}\right]}{\left(b^{2}-w^{2}\right)\left[(2-b)^{2}-w^{2}\right]}
$$

and consequently $G^{\prime}(w)$ does not vanish on $0<w<d$. We will see in Theorem 4 that if $\eta_{0}=\eta_{2}$ then for the extremal domain the slits are equidistant from the origin.

Example 3. With the additional restriction that $\eta_{1}=\eta_{2}$ we will show that in this case the slits on the boundary of the extremal domain are equidistant from the origin only when $\sigma_{k} \pi=2 k \pi / 5$. As in the previous two examples, we have from (2.9) that the extremal function $f(z)$ satisfies

$$
\left|f\left(e^{i \phi}\right)\right|^{2}=(1 / 16) y^{-\eta} 0\left|y-a_{1}\right|^{-\eta_{1}}\left|y-a_{2}\right|^{-\eta_{1}} \quad\left(y=\sin ^{2}(\phi / 2)\right),
$$

where by (2.12) $a_{1}$ and $a_{2}$ satisfy

$$
\begin{aligned}
& \eta_{1} / 2\left(a_{1}-1\right)+2 \eta_{1} /\left(a_{1}-a_{2}\right)+\left(\eta_{0}+\eta_{1}\right) / a_{1}=0 \\
& \eta_{1} / 2\left(a_{2}-1\right)-2 \eta_{1} /\left(a_{1}-a_{2}\right)+\left(\eta_{0}+\eta_{1}\right) / a_{2}=0 .
\end{aligned}
$$

The point $z=-1$ is mapped onto the tip of the slit along the ray $\arg w=\pi$. Also, $\exp \left(i \phi_{1}\right), \exp \left(-i \phi_{1}\right), \exp \left(i \phi_{2}\right)$ and $\exp \left(-i \phi_{2}\right)$ where $a_{k}=\sin ^{2}\left(\phi_{k} / 2\right)$ $(k=1,2)$ are the pre-images of the tips of the other slits on the boundary of $f(U)$. To show the slits are not equidistant from the origin unless $\sigma_{k} \pi=2 k \pi / n$ we examine the equation

$$
\left|f\left(e^{i \phi_{1}}\right)\right|^{2}\left|f\left(e^{i \phi_{2}}\right)\right|^{2}=|f(-1)|^{4}
$$

which is equivalent to

$$
\begin{gathered}
\left(y_{1} y_{2}\right)^{\eta}{ }^{\eta}\left|\left(y_{1}-a_{1}\right)\left(y_{2}-a_{2}\right)\right|^{\eta}{ }_{1}\left|\left(y_{1}-a_{2}\right)\left(y_{2}-a_{2}\right)\right|^{\eta} 1 \\
=\left(1-a_{1}\right)^{2 \eta_{1}}\left(1-a_{2}\right)^{2 \eta_{1}} .
\end{gathered}
$$


If we can show that (3.8) has only one solution for $\eta_{1}$ with $\eta_{1}>0$, we will have established our claim. Recall that $y_{1}$ and $y_{2}$ are solutions of $\eta_{0} / y+\eta_{1} /\left(y-a_{1}\right)+$ $\eta_{1} /\left(y-a_{2}\right)=0$ and consequently by (2.7) (and the fact that $\eta_{1}=\eta_{2}$ )

$$
\begin{aligned}
\left|\left(y_{1}-a_{1}\right)\left(y_{2}-a_{1}\right)\right| & =\left[\eta_{1} a_{1}\left(a_{2}-a_{1}\right) / 2\right], \\
\left|\left(y_{1}-a_{2}\right)\left(y_{2}-a_{2}\right)\right| & =\left[\eta_{1} a_{2}\left(a_{2}-a_{1}\right) / 2\right], \\
y_{1} y_{2} & =1 / 2 \eta_{0} a_{1} a_{2} .
\end{aligned}
$$

From these equations it follows that (3.8) is equivalent to

$$
\eta_{0}^{\eta}{ }^{0} \eta_{1}^{2 \eta}{ }^{1}\left(a_{1} a_{2}\right)^{\eta} 0^{+\eta} 1\left(a_{2}-a_{1}\right)^{2 \eta} 1=4\left[\left(1-a_{1}\right)\left(1-a_{2}\right)\right]^{2 \eta_{1}} \text {. }
$$

Since $\eta_{1}=\eta_{2}$, it follows from (2.13) that

$$
a_{1}+a_{2}=4 /\left(2+3 \eta_{1} / 2\right)
$$

Using this equation and the fact that $a_{1}$ and $a_{2}$ satisfy (3.7), it can also be shown that

$$
a_{1} \cdot a_{2}=4\left(1-\eta_{1} / 2\right) /\left(2+3 \eta_{1} / 2\right)\left(2+\eta_{1} / 2\right) .
$$

Using (3.10) and (3.11) we see after a short calculation that (3.9) is equivalent to

$$
3^{\eta}{ }^{1}\left(2+3 \eta_{1} / 2\right)^{\eta_{0}+\eta^{1}} 1\left(2+\eta_{1} / 2\right) \eta^{\eta}=\left[2 \eta_{0}\left(1-\eta_{1} / 2\right)\right]^{\eta_{0}}\left[64\left(1-\eta_{1} / 2\right)\right]^{\eta} 1
$$

If we denote the left-hand side of the previous equation by $L\left(\eta_{1}\right)$ and the right-hand side by $R\left(\eta_{1}\right)$ then we have $L(0)=R(0)$ and $L(4 / 5)=R(4 / 5), \eta_{1}=4 / 5$ corresponds to the case that $\sigma_{k} \pi=2 k \pi / 5$. Let $\phi\left(\eta_{1}\right)=\log L\left(\eta_{1}\right)-\log R\left(\eta_{1}\right)$. An easy calculation shows that $\phi\left(\eta_{1}\right)$ is a convex function of $\eta_{1}$ and hence $\phi\left(\eta_{1}\right)$ has $\eta_{1}=0$ and $\eta_{1}$ $=4 / 5$ as its only zeros which implies that (3.12) and hence (3.8) has only one solution on the interval $\eta_{1}>0$.

In the previous examples we have seen that if $n=3$ or 5 (and hence odd) the slits on the boundary of the extremal domain are equidistant from the origin only when $\sigma_{k} \pi=2 k \pi / n$ (with $n=5$ we had the restriction that $\eta_{1}=\eta_{2}$ ). For the case $n=4$, the slits were equidistant from the origin not only if $\sigma_{k} \pi=2 k \pi / 4$ but also whenever $\eta_{0}=\eta_{2}$. In the following theorem we prove a result for arbitrary even $n$ that includes this observation for $n=4$ as a special case.

Theorem 4. Let $\left\{\sigma_{k} \pi\right\}_{k=1}^{2 n}$ satisfy

$$
\begin{aligned}
\sigma_{2 k+1} \pi-\sigma_{2 k} \pi & =\beta \pi & (k=1, \cdots, n-1), \\
2 \pi+\sigma_{1} \pi-\sigma_{2 n} \pi & =\beta \pi, & \sigma_{1} \pi=1 / 2 \beta \pi
\end{aligned}
$$

and 


$$
\sigma_{2 k+2} \pi-\sigma_{2 k+1} \pi=\alpha \pi \quad(k=0,1, \cdots, n-1) .
$$

Then $\Pi_{k=1}^{2 n} l_{k} \geq 1 / 4\left(1 / 2 n \sigma_{1}\right)^{2 n \sigma_{1}}\left(1 /\left(2-2 n \sigma_{1}\right)\right)^{2-2 n \sigma_{1}}$. The slits on the boundary of the extremal domain are equidistant from the origin.

Proof. The conditions (3.13) and (3.14) imply that

$$
\begin{array}{rlrl}
\sigma_{k} \pi & =2 \pi-\sigma_{2 n-k+1} \pi & (k=1, \cdots, 2 n), \\
\alpha \pi+\beta \pi & =2 \pi / n, & & \\
\sigma_{k+2} \pi=\sigma_{k} \pi & =2 \pi / n & (k=1, \cdots, 2 n) .
\end{array}
$$

Since (3.15) holds, we have from Theorem 1 that there is an extremal domain, $D$, for this problem that is the complement of slits that are symmetric with respect to the real axis. From (3.17) we see that if we symmetrize $D$ using (1.3) with $\alpha_{k}=$ 1 and $\beta_{k}=2 k \pi / n$, we obtain a domain $D^{*}$ which is again symmetric with respect to the real axis and in addition satisfies the condition $l_{k+2}\left(D^{*}\right)=l_{k}\left(D^{*}\right)$ for $1 \leq k \leq$ $2 n-2$. That is, $D^{*}$ is the complement of slits along the rays $\arg w=\sigma_{k} \pi$ that are equidistant from the origin. By an argument similar to one used in the proof of Theorem 1 , it follows that $D^{*}$ is an extremal domain for this problem.

From (3.13) we have that $2 \sigma_{1} \pi=\beta \pi$ and from (3.16) that $n \sigma_{1} \pi<\pi$. Let $f(z)$ denote the function that maps $U$ onto the extremal domain of (2.10) with $\eta=2 n \sigma_{1}$. From the geometry of $D^{*}$ it follows that $g(z)=\left[f\left(z^{n}\right)\right]^{1 / n}$ maps $U$ onto $D$. Consequently,

$$
\prod_{k=1}^{2 n} l_{k}\left(D^{*}\right) \geq \frac{1}{4}\left[\frac{1}{2 n \sigma_{1}}\right]^{2 n \sigma_{1}}\left[\frac{1}{2-2 n \sigma_{1}}\right]^{2-2 n \sigma_{1}}
$$

and the proof is complete.

4. Final remarks. Considering the method of symmetrization introduced above and the way it was applied-the following question is natural: Let $0 \leq \theta_{1}<\theta_{2}$ $<\cdots<\theta_{n}<2 \pi$. Under what conditions on $S=\left\{\theta_{1}, \cdots, \theta_{n}\right\}$ do there exist linear transformations $H_{0}=\left\{L_{1}, \cdots, L_{n}\right\}, L_{j}(\theta)=\alpha_{j} \theta+\beta_{j}$, where $\alpha_{j}= \pm 1$ and $\beta_{j}$ is real, with the property that

$$
\bigcup_{k=1}^{n} L_{k}\left(\theta_{j}\right)=S
$$

for $j=1, \ldots, n$ ?

In order to answer the question we construct the set $H$ of linear transformations $Q$ with the properties

(i) $Q(S)=S$,

(ii) $Q \theta=\alpha_{j} \theta+\beta_{j}\left(\alpha_{j}= \pm 1, \beta_{j}\right.$ real $)$. 
$H$ is a group under composition as is easily shown. It is also clear that $H_{0} \mathrm{CH}$.

We will call $Q$ a translation if $\alpha=1$ and an inversion if $\alpha=-1$. A translation will be denoted by $T$ and an inversion by $P$.

Using (i) and (ii) it is easy to see that the set of translations $\hat{H}$ in $H$ forms a cyclic subgroup of $H$. Either $H=\hat{H}$ or, for any fixed inversion $P$ in $H, H=\hat{H}$ $\cup \hat{H} P$. Indeed, if $T \in H$ is a translation it belongs to $\hat{H}$ and if $P_{1} \in H$ is an inversion $P_{1}=X P$ implies $X$ is a translation, and so $P_{1} \in \hat{H} P$. Let $T$ denote a generator of $\hat{H}$ and $S_{1}=\bigcup_{k} T^{k} \theta_{1}$. There are two cases: Either $S_{1}=s$ in which case the $\theta_{j}$ are equally spaced, or $S_{1}$ is a proper subset of $S$. For the latter case let $\theta_{j} \in S \backslash S_{1}$. By (4.1), $\theta_{j}=Q \theta_{1}$ for some $Q \in H_{0}$. Since $\theta_{j} \notin S_{1}, Q \notin \hat{H}$ and so $Q=T^{k} P$ for some $k$. Thus $\theta_{j} \in \bigcup_{k} T^{k} P \theta_{1}$ and hence $S=\left\{\bigcup_{k} T^{k} \theta_{1}\right\} \cup\left\{\bigcup_{k} T^{k} P \theta_{1}\right\}$ which is the configuration of the $\theta_{k}$ in Theorem 4 .

We may summarize by saying that only two configurations of the $\theta_{k}$ are possible in order that (4.1) hold. Either we have equally spaced angles or we have the configurations of Theorem 4.

Based on the examples of $\$ 3$ and the information of this section, we pose an open problem which seems to be interesting. Do there exist configurations of the $\theta_{k}$ other than that of Theorem 4 and when the $\theta_{k}$ are equally spaced such that the slits of the boundary of the extremal domain are equidistant from the origin?

\section{BIBLIOGRAPHY}

1. D. Aharonov and W. E. Kirwan, A method of symmetrization and applications. I, Trans. Amer. Math. Soc. 163 (1972), 369-377.

2. G. M. Goluzin, Geometric theory of functions of a complex variable, GITTL, Moscow, 1952; English transl., Transl. Math. Monographs, vol. 26, Amer Math. Soc., Providence, R. I., 1969. MR 15, 112; MR $40 \# 308$.

3. W. Hayman, Multivalent functions, Cambridge Tracts in Math. and Math. Phys., no. 48, Cambridge Univ. Press, Cambridge, 1958. MR 21 \#7302.

4. M. Klein, Estimates for the transfinite diameter with applications to conformal mapping, Pacific J. Math. 22 (1967), 267-279. MR 37 \#1577.

$5 \mathrm{M}$. Marcus, Transformations of domains in the plane and applications in the theory of functions, Pacific J. Math. 14 (1964), 613-626. MR 29 \#2382.

6. I. Schur, Über die Verteilung der Wurzeln bei gewissen algebraischen Gleichungen mit ganzzahligen Koeffizienten, Math. Z. 1 (1918), 377-402.

7. T. J. Stieltjes, Sur quelques théorèmes d'algèbre, Comptes Rendus 100 (1885), 439-440.

8. G. Szegö, On a certain kind of symmetrization and its applications, Ann. Mat. Pura Appl. (4) 40 (1955), 113-119. MR 17, 1074.

DEPARTMENT OF MATHEMATICS, TECHNION-ISRAEL INSTITUTE OF TECHNOLOGY, HAIFA, ISRAEL

DEPARTMENT OF MATHEMATICS, UNIVERSITY OF MARYLAND, COLLEGE PARK, MARYLAND 20742 